Trauma Berufskrankh 2008 · 10

[Suppl 1]:107-111

DOI 10.1007/s10039-007-1276-6

Online publiziert: 30 . August 2007

(c) Springer Medizin Verlag 2007

\author{
R. Beickert \\ BG-Unfallklinik, Murnau
}

\title{
Zahlen und Fakten zur BGSW in Bayern und Sachsen
}

Die erneute stationäre Behandlung

Der Begriff „Berufsgenossenschaftliche Stationäre Weiterbehandlung" (BGSW) wurde 1991 in das berufsgenossenschaftliche Heilverfahren eingeführt. Er sollte in Anlehnung an die Bezeichnung „Anschlussheilbehandlung “ der Rentenversicherungsträger und Krankenkassen - für eine stationäre Rehabilitationsbehandlung verwendet werden, die sich an die Akutversorgung eines durch Arbeitsunfall Verletzten anschließt.

Seit 01.01.2006 werden die Indikationsstellung und die Inhalte der BGSW von der „Handlungsanleitung zur Verordnung, Durchführung und Qualitätssicherung der Physiotherapie - Physikalischen Therapie, Erweiterten Ambulanten Physiotherapie (EAP), Berufsgenossenschaftlichen Stationären Weiterbehandlung (BGSW) und Sonstigen stationären Maßnahmen“ des ehemaligen Hauptverbands der Gewerblichen Berufsgenossenschaften (HVBG) bestimmt. Die Realisierungsmöglichkeit der sehr umfangreichen Inhalte wurde von den Leistungserbringern sehr kritisch gesehen, da sie auf der Basis der Verträge zwischen den Landesverbänden und den Kliniken nicht umgesetzt werden konnten. Somit kann die Handlungsanleitung zunächst nur als Empfehlung verstanden werden, die mit den Realisierungsmöglichkeiten noch abgeglichen werden muss.

Als neue Behandlungsform wurde in die Rehabilitation Unfallverletzter die „Sonstige stationäre Maßnahme" eingeführt. Sie steht in der Hierarchie der möglichen Übungsbehandlungen über der BGSW, und es wurde ausdrücklich erläutert, dass diese Behandlungsformen überwiegend in den BG-Unfallkliniken stattfinden sollen.
Unfallverletzter im Rahmen des Rehabilitationsmanagements der Berufsgenossenschaften geht ebenfalls weit über die BGSW als Anschlussbehandlung hinaus. Sie ist inhaltlich als komplexe stationäre Rehabilitation mit der besonderen Zielsetzung der beruflichen Reintegration zu verstehen. Gekennzeichnet ist die „RehaBGSW“ durch eine besonders enge $\mathrm{Zu}$ sammenarbeit zwischen den Mitarbeitern der Berufsgenossenschaften und den Ärzten der Rehabilitationskliniken an der Schnittstelle zwischen medizinischer Behandlung und den Maßnahmen zur Teilhabe am Arbeitsleben.

\section{Definition BGSW}

Sie beinhaltet die nach Abschluss der Akutbehandlung in zeitlichem Zusammenhang stehenden medizinisch indizierten stationären Leistungen zur medizinischen Rehabilitation, insbesondere die intensive Übungszogener Abklärung).

Sie umfasst den Zeitraum, in dem bei schweren Verletzungen des Stütz- und Bewegungsapparats und des zentralen und peripheren Nervensystems zur Optimierung des Rehabilitationserfolgs ambulante Leistungen zur medizinischen Rehabilitation nicht ausreichen oder nicht möglich bzw. nicht durchführbar sind.

\section{Indikation zur BGSW}

Generell können 3 Hauptgruppen unterschieden werden:

- Schwere der Verletzung behandlung (ggf. unter Einschluss arbeitsbe-
- Komplikationsverläufe (Infektion, Pseudarthrose, CRPS)

- Andere Gründe (soziale Umstände, Standortprobleme)

Die wesentliche Voraussetzung von Seiten des Patienten ist die Rehabilitationsfähigkeit. Dieser Umstand ist in den zurückliegenden Jahren zunehmend unberücksichtigt geblieben. Es ist unbestreitbar, dass frisch verletzte Unfallpatienten in die BGSW-Klinik verlegtwerden müssen, wenn sieverletzungsbedingt noch nicht ausreichend mobil sind und sich nicht selbst versorgen können. Nach vielen rekonstruktiven Folgeeingriffen macht eine stationäre Rehabilitation aber erstdann Sinn, wenn bestimmteEntlastungsoder Ruhigstellungszeiten abgelaufen sind (z.B. rekonstruktive Eingriffe am Knochen und den großen Gelenken wie Hüft- und Knie-TEP). Die politische gewollte Verkürzung der Liegezeiten in den „Akut"-kliniken und die damit verknüpfte Abrechnung über DRG führen zwangsläufig dazu, dass die Rehabilitationskliniken Aufgaben übernehmen müssen, für die sie nicht eingerichtet sind und deren Kosten durch die Vertragstarife nicht abgedeckt sind. Zudem wird das Budget für die Rehabilitationsbehandlung zu früh aufgebraucht, was zwangsläufig zu einer Verlängerung der Gesamtbehandlungsdauer und möglicherweise auch zu einem ungünstigen Endergebnis führt.

Keine Indikationen für eine BGSW sind laut Handlungsanleitung

- Monoverletzungen und

- Fehlende Belastbarkeit für eine komplexe Nachbehandlung 
Dieser Passus der Handlungsanleitung ist missverständlich. Es sollte wohl zum Ausdruck kommen, dass z.B. der unkomplizierte Unterschenkelbruch oder die körpernahe Oberarmfraktur keiner stationären Rehabilitation bedürfen und dass auch dann ein stationäres Verfahren nicht eingeleitet werden soll, wenn der Zustand des Verletzten eine umfassende Übungsbehandlung noch nicht zulässt. Der Begriff „Monoverletzung“ ist zu unscharf, um in jedem Fall eine stationäre Rehabilitation auszuschließen. Auch die Schienbeinkopf- oder die Fersenbeinfraktur sind Monoverletzungen, die jedoch in vielen Fällen eine aufwändige Nachbehandlung erforderlich machen.

Sonstige Indikationen für BGSW können soziale und organisatorische Gründe sein:

- Wenn eine EAP-Einrichtung nicht in Wohnortnähe liegt.

- Wenn verschiedene medizinische Behandlungen an unterschiedlichen Standorten liegen, sodass lange Wegstrecken zurückgelegt werden müssen.

- Wenn so genannte „weiche Faktoren“ patientenseits vorliegen, die eine konzentrierte und effektive ambulante Behandlung nicht möglich machen.

\section{Behandlungsinhalte und Leistungsspektrum der BGSW}

Anders als in der vorausgehenden Handlungsanleitung von 1991 sind jetzt neben den Behandlungsinhalten, die sich nicht wesentlich geändert haben, Vorgaben zum Leistungsumfang zu berücksichtigen.

Die Behandlungsinhalte der BGSW sind weitgehend mit denen der EAP identisch. Gefordert werden:

- Physiotherapeutische/krankengymnastischer Therapie

- Physikalische Therapie

- Medizinische Trainingstherapie

- Ergotherapie

- Psychosoziale Betreuung

Bei Verletzungen des zentralen/peripheren Nervensystems werden zusätzlich verlangt:

- Logopädie

- Neuropsychologische Therapie

- Psychotherapie
- Soziotherapie

- Angehörigenbetreuung/-beratung

- Rehabilitationspflege

Der Leistungsumfang der BGSW umfasst therapeutische und arbeitsbezogene Maßnahmen, wobei die Behandlungsdauer als Mindestanforderung deklariert wird:

- 3 h Einzelbehandlung, z. B.

- 30 min Physiotherapie als Einzelbehandlung

- 60 min medizinische Trainingstherapie (MTT, MAT)

- 60 min Ergotherapie als Einzeltherapie

- 30 min physikalische Therapie

Darüber hinaus werden jetzt arbeitsbezogene Maßnahmen definiert, die den Übergang in die berufliche Rehabilitation bahnen sollen:

- Eine unter medizinischen Rahmenbedingungen vorgenommene Belastungserprobung und Arbeitstherapie unter Zugrundelegung der Tätigkeit, die der Patient bisher im Betrieb durchgeführt hat mit der Zielrichtung:

a) Feststellung des Leistungsbildes hinsichtlich der Arbeitsfähigkeit

b) Feststellung des Leistungsbildes hinsichtlich der weiteren Behandlungsbedürftigkeit

c) Feststellung des Leistungsbildes hinsichtlich der evtl. anstehenden Leistungen zur Teilhabe am Arbeitsleben

- Eine medizinisch-psychologische Beurteilung hinsichtlich der notwendigen Berufsqualifizierungsmaßnahmen und in welche Richtung diese evtl. gehen könnten

Als Methoden der Prüfung, ob Maßnahmen der Teilhabe am Arbeitsleben notwendig werden, stehen zur Verfügung:

a) Belastungserprobung und Arbeitstherapie

b) Evaluation der funktionellen Leistungsfähigkeit (EFL)

c) Arbeitsplatzsimulation (ERGOS)

d) Instrument zur Integration von Menschen mit Behinderungen in die Arbeitswelt (IMBA)

e) Erwerbsbezogene Ergotherapie (EET)
Die Handlungsanleitung empfiehlt also das gesamte Spektrum der medizinischen Rehabilitation und deren Assessmentverfahren in einer Phase der Behandlung, die sich definitionsgemäß an die Akuttherapie anschließen soll. Darin besteht wohl das grundlegende Missverständnis, denn der Leistungsumfang und die arbeitsbezogenen Maßnahmen sind in der Frühphase der Rehabilitation noch nicht notwendig. Sie sind dann erforderlich, wenn im Rahmen der Heilverfahrenssteuerung oder des Rehabilitationsmanagements eine Komplikation, eine Behandlungsverzögerung oder gar ein Behandlungsstillstand erkannt werden. Inhaltlich entspricht der oben skizzierte Leistungsumfang also nicht der BGSW, sondern der komplexen stationären Rehabilitation (s. unten).

Die neue Handlungsanleitung enthält erstmals Hinweise zur Qualitätssicherung der BGSW. Dazu dient in erster Linie das umfangreich gewordene Berichtswesen, das den Nachweis der erbrachten Leistungen im Detail vorsieht. Ob weitergehende Qualitätssicherungsmaßnahmen sinnvoll sind, muss zunächst offen bleiben. Es gibt verschiedene Fragebögen, die die Versicherten zu Beginn und zum Ende der Behandlung ausfüllen können, sodass neben objektiven Messungen der Funktion (des Funktionsgewinns) auch Informationen über die Befindlichkeit und den subjektiven Erfolg möglich sind. Beispiele für derartige Assessmentverfahren sind:

- Western Ontario and MacMasters Universities (WOMAC) Arthroseindex

- Disabilities of the Arm, Shoulder and Hand Score (DASH)

- Funktionsfragebogen-Hannover-Rücken (FFb-H-R)

- Short-Form Health Survey (SF 36)

- Gesundheitsfragebogen EQ-5D

\section{Sonstige stationäre Maßnahmen}

Sie unterscheiden sich von der BGSW hinsichtlich ihrer Indikation und ihrem Behandlungsumfang. Nachdem aber Letzterer nicht weiter definiert ist, kann davon ausgegangen werden, dass der komplexen stationären Rehabilitation (KSR) zumindest der oben beschriebene Leistungsumfang der BGSW zugrunde zu legen ist. Sonstige stationäre Maßnahmen 
sollen - aufgrund des besonderen Auftrags - überwiegend in den BG-Kliniken durchgeführt werden. Indikationen für die KSR sind:

\section{- Mehrfachverletzungen an Extremi- täten und Rumpf \\ - Querschnittlähmungen \\ - Schädel-Hirn-Verletzungen \\ - schwere Brandverletzungen \\ - schwere Handverletzungen \\ - Komplikationen (Osteitis, Dystro- phiesyndrom, usw.) \\ - Gliedmaßenverlust zur prothetischen Versorgung}

Darüber hinaus kommen für eine komplexe stationäre Rehabilitation in einer BG-Klinik auch alle anderen Fälle in Betracht, bei denen eine UnverhältnismäBigkeit zwischen Art und Schwere der Verletzung und der Dauer des Heilverfahrens oder eine Problematik bei der Teilhabe am Arbeitsleben erkennbar sind. Die Indikationen beinhalten zunächst wieder die Regelindikation der BGSW, wobei die Schwere der Verletzung (Rückenmark, Brand-, Handverletzung) ohnehin die Versorgung in einer speziellen Einrichtung erforderlich macht. Darüber hinaus gibt es Verlaufsformen und Komplikationen (Osteitis, Fasziitis, Dystrophiesyndrom), die grundsätzlich in BG-Kliniken behandelt werden sollten, um das Rehabilitationspotenzial möglichst umfassend ausschöpfen zu können. Dazu kommt der „ungewöhnliche“ Behandlungsverlauf, der dann vorliegt, wenn zwischen Verletzungsart und -schwere sowie Dauer der Behandlung eine erhebliche Diskrepanz besteht, wenn ein chronisches Schmerzsyndrom entstanden ist oder psychische Störungen aufgetreten sind, die mit dem Unfall im Zusammenhang stehen könnten und/oder die Behandlung oder die Dauer der Arbeitsunfähigkeit beeinflussen.

\section{BGSW in Bayern und Sachsen 2005}

2005 wurden in Bayern 2574, in Sachsen 1165 BGSW-Verfahren im orthopädisch/ unfallchirurgischen Fachgebiet durchgeführt, dazu kommen 579 Verfahren der neurologischen Rehabilitation (350 in Bayern und 229 in Sachsen) Aus der jeweiligen Behandlungsdauer kann man eine durchschnittliche Verweildauer er- rechnen. Diese betrug im orthopädisch/ unfallchirurgischen Fachgebiet in Bayern 26,7 und in Sachsen 28,9 Tage. Über die Zahl der Berechnungstage und das klinikspezifische Entgelt lassen sich die Gesamtkosten und die Kosten/Fall errechnen (• Tab. 1).

Die Entgelt-Kosten-Diskussion entzündet sich an der Tatsache, dass bei einem durchschnittlichen Entgelt pro Tag von 118,35 EUR die neue Handlungsanleitung BGSW nicht umgesetzt werden kann. Ein Physiotherapeut kostet den Arbeitgeber z. B. 20,33 EUR/Arbeitsstunde, d. h., wenn arbeitstäglich mindestens(!) 3 h Einzeltherapie gefordert werden, würde das Tagesentgelt schon allein von der Physiotherapie zur Hälfte aufgebraucht werden. Wenn die Handlungsanleitung Bestand haben und auch umgesetzt werden soll, ist dies zu dem bisherigen BGSW-Tarif nicht möglich.

Der hohe personelle und materielle Aufwand, den eine komplexe stationäre Rehabilitation erfordert, kommt im so genannten BGSW-Tarif der BG-Unfallklinik Murnau zum Ausdruck: Neben dem Basistarif von 138,89 EUR, der für alle Abteilungen gleich ist und sich aus der Infrastruktur der Klinik ableitet, beträgt der Behandlungstarif der Rehabilitationsstation 170,58 EUR. Darin enthalten sind alle Aufwendungen, die pflegerisch und ärztlich ausgelöst werden, also das gesamte Spektrum der physikalischen Therapie, die Aufwendungen für Diagnostik, ärztliche Behandlung einschließlich Schmerztherapie, psychologische Behandlung und Psychotherapie. Der Gesamtaufwand pro Tag beträgt 309,47 EUR, und bei einer durchschnittlichen Verweildauer von 29 Tagen ergibt dies einen Durchschnittspreis für die komplexe stationäre Rehabilitation (KSR) von 8974,63 EUR. Die Kosten liegen also 3-mal so hoch wie die durchschnittlichen Kosten einer BGSW in Bayern oder Sachsen.

Die Entgelt-Kosten-Diskussion hat aber, wenn man die gesamten Behandlungskosten einer Verletzung und deren Folgen beleuchtet, mehrere Aspekte. Anders als die Krankenkassen sind die Träger der gesetzlichen Unfallversicherung verpflichtet, für die Folgen einer Verletzung aufzukommen. Das lenkt den Schwerpunkt der Bemühungen der Unfallversi-
Trauma Berufskrankh 2008 · 10

[Suppl 1]:107-111

DOI 10.1007/s10039-007-1276-6

๑) Springer Medizin Verlag 2007

\section{R. Beickert \\ Zahlen und Fakten zur BGSW in Bayern und Sachsen}

\section{Zusammenfassung}

Die berufsgenossenschaftliche stationäre Weiterbehandlung (BGSW) stand bisher in engem Zusammenhang mit der Akutbehandlung und war der Anschlussheilbehandlung vergleichbar. In jüngster Zeit hat jedoch der Umfang der Therapie erheblich zugenommen, zudem wurden die zur BGSW zugelassenen Einrichtungen durch die neue Handlungsanleitung mit neuen Aufgaben bedacht, was beides mit höheren Kosten einhergeht. Im vorliegenden Beitrag werden die Indikation, die Behandlungsinhalte und das Leistungsspektrum der BGSW vorgestellt und anhand von Zahlen der Bundesländer Bayern und Sachsen die Kostensituation diskutiert. Abschließend wird auf die Konzepte der BG-Kliniken zur Umsetzung der neuen BGSW eingegangen.

\section{Schlüsselwörter}

Berufsgenossenschaftliche stationäre Weiterbehandlung · BGSW - Anschlussheilbehandlung $\cdot$ Handlungsanleitung $\cdot$ Kosten

\section{Facts and figures on BGSW in Bavaria and Saxony}

\section{Abstract}

Further in-patient treatment approved by the employers' liability insurance associations (BGSW), which has so far been closely connected with acute treatment and comparable to immediate retreatment, is in a state of flux. Recently, therefore the range of treatments has been considerably extended, in addition to which the institutions approved for application of BGSW now have new obligations imposed by the new management guidelines, all of which means higher costs. In the present paper the indications for BGSW, and content of the treatment and the range of benefits are presented and the economics of the situation is discussed with reference to figures recorded in Bavaria and Saxony. The employers' liability insurance associations' projected methods of implementing the new BGSW are then discussed in some detail.

\section{Keywords}

Further in-patient treatment approved by the employers' liability insurance associations . BGSW $\cdot$ Immediate retreatment . Management guideline $\cdot$ Costs 


\begin{tabular}{|c|c|}
\hline Bereich & Kosten [EUR] \\
\hline Orthopädie/Unfallchirurgie & 11.967 .455 \\
\hline Kosten pro Fall in Bayern & 3156 \\
\hline Kosten pro Fall in Sachsen & 3021 \\
\hline
\end{tabular}

Tab. 2 Kosten der Behandlung einer Fersenbeinfraktur

\begin{tabular}{|ll}
\hline $\begin{array}{l}\text { Maßnahme } \\
\text { Sofortmaßnahmen/ } \\
\text { Transport }\end{array}$ & $\begin{array}{l}\text { Kosten [EUR] } \\
500\end{array}$ \\
\hline Operation & 1800 \\
\hline Stationärer Aufenthalt & 6208 \\
\hline Ambulante Behandlung & 5041 \\
\hline Rehabilitation & 3300 \\
\hline Ergänzende Heilbehandlung & 3750 \\
\hline Verletztengeld & 8416 \\
\hline $\begin{array}{l}\text { Durchschnittliche } \\
\text { monatliche Rente }\end{array}$ & 367 \\
\hline Gesamtrente bis 65 & 88.080 \\
\hline Gesamtkosten & 117.095 \\
\hline Behandlungskosten & 29.015 \\
\hline
\end{tabular}

cherungsträger auf die Unfallverhütung (Prävention) und die Behandlung mit allen geeigneten Mitteln (Rehabilitation) mit dem Ziel, die Folgen, die sich für den Versicherten in einer Minderung der Erwerbsfähigkeit darstellen, so gering wie möglich zu halten.

Beispielhaft sind in $\mathbf{0}$ Tab. 2 die Kosten der Behandlung einer Fersenbeinfraktur auf der Basis von Zahlen, die über den HVBG zu erfahren waren (Infobox 1), dargestellt:

Zugrunde liegen Daten von etwa 2400 neuen Verletzungen/Jahr, einem Durchschnittsalter der Verletzten von 45,5 Jahren und einer durchschnittlichen MdE von $24 \%$

Die Behandlungskosten machen 24,8\% der Gesamtkosten aus, die Rehabilitation $11,4 \%$ der Behandlungskosten oder 2,8\% der Gesamtkosten.

\section{Konzepte der BG-Kliniken}

Die berufsgenossenschaftlichen Unfallkliniken haben sich den veränderten Rahmenbedingungen, die sich aus der neuen Handlungsanleitung zur Übungsbehandlung ergeben, gestellt. Sie haben Konzepte entwickelt, die zu jedem Zeitpunkt des Heilverfahrens den Einsatz des jeweils am besten geeigneten Instruments erlauben.
Die „klassische“ BGSW obliegt in erster Linie den zugelassenen Einrichtungen in Bayern und Sachsen. Bezüglich der Erlössituation für diese Kliniken bleibt abzuwarten, wie der Verwaltungsausschuss „Heilverfahren"desDGUVhinsichtlich der Inhalte der BGSW entscheiden wird. Wird an den Inhalten der „Handlungsanleitung“ festgehalten, müssen die Verträge mit den zugelassenen Kliniken überarbeitet werden.

BGSW und Wiederholungsrehabilitation sind Instrumente des D-Arzt-Verfahrens, sie werden per Formblatt F 2150 verordnet. Einer besonderen Begründung bedarf im Genehmigungsverfahren lediglich die Wiederholungsrehabilitation.

Die Sonderstellung der BG-Kliniken ist darin begründet, dass sie schon jetzt den Anforderungen der Handlungsanleitung gerecht werden, aber nur im Sinne der „komplexen stationären Rehabilitation“. Die Indikationsstellung zur KSR erfolgt in der Regel anlässlich einer Heilverfahrenskontrolle oder im Rahmen des Rehabilitationsmanagements der Berufsgenossenschaft. Die Indikation und Verordnung einer KSR bedürfen also einer besonderen unfallmedizinischen Kompetenz.

\section{Rehabilitationsabklärung}

Mehrere Unfallkliniken bieten dieses Konzept als übergeordnete Heilverfahrenskontrolle an. Die Erfahrung lehrt, dass die bisherigen Instrumente der Heilverfahrenskontrolle in vielen Fällen nicht ausreichten, insbesondere bei der Straffung und Beschleunigung des Heilverfahrens. Rehabilitationsabklärung hat das Ziel, eine sachliche und kompetente Aussage dazu zu erhalten, ob aus medizinischer Sicht weitere Rehabilitationsbemühungen Sinn machen oder ob ein Behandlungsende festzustellen ist, womit sogleich Berufshilfemaßnahmen eingeleitet werden könnten. In diese Rehabilitationsabklärung sind Unfallchirurgen, Psychologen, Physiotherapeuten und Ergotherapeuten eingebunden, wobei jede Berufsgruppe zu der spezifischen Fragestellung: „besteht noch medizinischer Rehabilitationsbedarf" abschließend in einem gemeinsamen Protokoll Stellung nimmt. Voraussetzung für eine kompetente Sachaussage zum Stand der medizinischen Rehabilitation sind umfassende reprodu- zierbare Messungen des körperlichen und geistigen Leistungsvermögens. Deshalb ist es notwendig, in die Bestandsaufnahme Testverfahren einzuführen, die Aussagen zur funktionellen und psychischen Leistungsfähigkeit zulassen. Testverfahren stehen deshalb im Mittelpunkt der Evaluation, die nach Erstellung des Prüfberichts Grundlage eines dreiseitigen Übereinkommens zwischen Arzt, Versichertem und Berufshelfer sein kann.

Ergibt die Abklärung, dass Rehabilitationspotenzial besteht, kann die medizinische Behandlung zielorientiert geplant und umgesetzt werden, wobei die BG-Kliniken nur dann in die Therapie eingebunden werden, wenn andernorts das gleiche Ziel nicht erreicht werden kann.

Die BG-Kliniken sind somit gefordert, Behandlungsformen anzubieten, die in besonders schwierigen Fällen erforderlich sind. Das kann im Sinne der Handlungsanleitung von der Verletzungsschwere bestimmt sein, z. B. bei Hand- und bei Rückenmarkverletzungen, aber auch von einem komplizierten Behandlungsverlauf diktiert sein wie bei einem chronisch regionalen Schmerzsyndrom oder einer Schmerzkrankheit. Schließlich können BG-Kliniken eine arbeitplatznahe Rehabilitation anbieten unter dem Aspekt, dass jede spezifische berufliche Tätigkeit spezielle körperliche und geistig-seelische Anforderungen an den Versicherten stellt.

\section{Komplexe stationäre Rehabilitation}

Konkret werden an der BG-Unfallklinik Murnau (wie auch an anderen BG-Kliniken) 3 Konzepte angeboten:

- Arbeitsplatzbezogene Rehabilitation

- Interdisziplinäre Schmerztherapie

- Rehabilitation nach Psychotrauma

Während Erstere in der Rückkehr zum Arbeitsplatz ein klar definiertes Ziel hat, das eine zeitliche Festlegung der Behandlungsdauer nicht erlaubt, können die interdisziplinäre Schmerztherapie und die Rehabilitation nach Psychotrauma nur eine Anstoßbehandlung sein, die wohnortnah fortgesetzt wird, mit der Maßgabe, dass der Versicherte über eine entsprechende Spezialambulanz an die Klinik gebunden bleibt, um auf Störungen des Heilverlaufs sofort reagieren zu können. 


\section{Arbeitsplatzbezogene Rehabilitation}

Für sie stehen alle Instrumente zur Verfügung, die in der Handlungsanleitung als Prüfmethoden bezeichnet sind:

- IMBA: Instrument zur Integration von Menschen mit Behinderungen in die Arbeitswelt

- EFL: Evaluation der funktionellen Leistungsfähigkeit nach Isernhagen

- ERGOS: Arbeitsplatzsimulationsgerät

- EET: erwerbsbezogene Ergotherapie

Diese Instrumente erlauben eine objektive Messung der körperlichen Leistungsfähigkeit, um das Rehabilitationspotenzial auszuloten und ein erstes positives Leistungsbild zu erstellen.

\section{Interdisziplinäre Schmerztherapie}

Sie erfordert - wie der Name sagt - eine fachübergreifende Zusammenarbeit von Ärzten verschiedener Fachrichtungen, Psychologen, Physiotherapeuten und Ergotherapeuten unter der Leitung eines speziell weitergebildeten Arztes (meist Anästhesisten). Im Vorfeld spielt die Prävention einer Schmerzkrankheit eine ganz entscheidende Rolle. Deshalb muss der Blick dahingehend geschult werden, zu erkennen, ob über die natürliche Schmerzhaftigkeit einer Verletzung, einer Operation oder eines Unfallfolgeschadens hinaus eine Chronifizierung droht.

\section{Rehabilitation nach Psychotrauma}

Die Behandlung des Psychotraumas ist Neuland für die Unfallkliniken. Schon der Begriff "psychische Verletzung" ist schwer zu definieren. Unfallchirurgen oder Orthopäden haben nicht die Kompetenz, einen seelisch/geistigen Körperschaden zu definieren, zu erkennen und zu behandeln, somit müssen Psychiater, Psychologen und Psychotherapeuten in das berufsgenossenschaftliche Heilverfahren eingebunden werden.

Im Rahmen einer stationären oder teilstationären Rehabilitation steht zunächst die Diagnosestellung im Vordergrund. Erst dann kann - wiederum interdisziplinär und häufig eng verbunden mit der Schmerztherapie - das Behandlungsziel festgelegt werden, wobei die Rehabilitation der physischen Körperschäden den individuellen Umständen angepasst werden muss.
Prüfmethoden, wie sie für die arbeitsplatzbezogene Rehabilitation angemessen sind, sind in der Schmerztherapie und in der Behandlung psychischer Unfallfolgen nur bedingt einsetzbar. Hier können die Testpsychologie und die Neuropsychologie kompetente Aussagen zur Leistungsfähigkeit machen.

\section{Resümee}

Die BG-Kliniken machen ihre Leistungsangebote transparent. Die Unfallklinik Murnau hat auf ihrer Homepage (Infobox 1) ein BG-Portal eingerichtet, das allgemein zugänglich ist, in erster Linie aber Informationen für die Mitarbeiter der berufsgenossenschaftlichen Verwaltungen enthält. Unter der Zeile „Reha-behandlungen“"werden die Rehabilitationskonzepte detailliert dargestellt und können als pdf-Datei eingesehen und geladen werden.

\section{Fazit}

Die BGSW unterliegt einem Wandel. Während sie bisher nahe an der Akutbehandlung lag und der Anschlussheilbehandlung der Rentenversicherungsträger glich, werden durch die neue Handlungsanleitung die zur BGSW zugelassenen Einrichtungen mit neuen Aufgaben bedacht, darüber hinaus hat der Umfang der Therapie erheblich zugenommen. Es liegt auf der Hand, dass größere Aufgaben auch mit höheren Kosten verbunden sind.

Sonstige stationäre Maßnahmen sind Behandlungen, die - wie bisher - den BGKliniken vorbehalten bleiben.

Die Anforderungen an die BG-Kliniken sind enorm gewachsen. Im Rahmen des Rehabilitationsmanagements bieten sie eine Rehabilitationsabklärung unter Einsatz bewährter Assessmentverfahren an. Chronisch schmerzkranke Patienten bedürfen einer interdisziplinären Betreuung, sie sollten nach der stationären Phase über Ambulanzen an die Klinik gebunden bleiben. Die Einbindung von Schmerztherapeuten und Psychologen in das berufsgenossenschaftliche Heilverfahren ist unter dem Aspekt einer umfassenden ganzheitlichen Behandlung von Unfallfolgen nicht mehr wegzudenken.

\section{Internetlinks}

- Homepage der BG-Unfallklinik Murnau: http://www.bgu-murnau.de

- Information zu Behandlungskosten: http://www.wdr.de/tv/q21/875.0.phtml

\section{Korrespondenzadresse}

\section{Dr. R. Beickert}

BG-Unfallklinik Murnau,

Prof. Küntscher-Straße 8, 82418 Murnau

beickert@bgu-murnau.de

Interessenkonflikt. Der korrespondierende Autor gibt an, dass kein Interessenkonflikt besteht. 